ournal of

\title{
Brachiocephalic Vessels Ultrasound Index of Arterio- venous Ratio (IAVR) and Headache as a Diagnostic Tool for Intracranial Venous Stasis in Arterial/Venous Ischemic Stroke's Differentiation
}

Semenov Stanislav ${ }^{*}$, Yurkevich Elena ${ }^{1}$, Semenov Aleksandr ${ }^{2}$, Korotkevich Alexey ${ }^{1}$ and Kokov Aleksandr ${ }^{1}$

${ }^{1}$ Research Institute for Complex Issues of Cardiovascular Diseases, Kemerovo, Russian Federation, Russia

${ }^{2}$ Praxis Wolfgang Theobald Facharzt für Radiologie, Saarlouis, Germany

Correspondence to:

Semenov Stanislav

Research Institute for Complex Issues of

Cardiovascular Diseases, Kemerovo

Russian Federation, Russia

Tel: $+7(3842) 341710$

E-mail:dr_semenov_s@mail.ru

Received: December 18, 2019

Accepted: January 23, 2020

Published: January 25, 2020

Citation: Stanislav S, Elena Y, Aleksandr S, Alexey K, Aleksandr K. 2020. Brachiocephalic Vessels Ultrasound Index of Arteriovenous Ratio (IAVR) and Headache as a Diagnostic Tool for Intracranial Venous Stasis in Arterial/Venous Ischemic Stroke's Differentiation. J Neuroimaging Psychiatry Neurol 5(1): 1-5.

Copyright: () 2020 Stanislav et al. This is an Open Access article distributed under the terms of the Creative Commons Attribution 4.0 International License (CC-BY) (http://creativecommons. org/licenses/by/4.0/) which permits commercial use, including reproduction, adaptation, and distribution of the article provided the original author and source are credited.

Published by United Scientific Group

\begin{abstract}
Summary: Identification of signs of intracranial venous stasis using ultrasound methods in combination with determination of headache intensity according to the visual-analogue scale allows us to suspect the venous genesis of ischemic stroke before using neuroimaging methods, which can contribute to the selection of adequate therapy and to improve the prognosis and long-term outcomes of the disease.
\end{abstract}

Objective: To study the prognostic value of a complex of clinical demographic and instrumental indicators for developing a model for early differential diagnosis of ischemic stroke of arterial and venous origin.

Material and Methods: Examined 124 patients with ischemic stroke: 22 with venous stroke due cerebral venous sinus thrombosis (VIS), $53.5 \pm 16.7 \mathrm{y}$ and 102 with atherothrombotic stroke (AIS), $68.3 \pm 12.1 \mathrm{y}$ which were verified by native CT; CT-angiography, PCT (perfusion CT: CBF, CBV, MTT), brachiocephalic vessels ultrasound index of arteriovenous ratio (IAVR) and transcranial duplex scanning for all. IAVR was obtained due duplex scanning of carotid common arteries (CCA) and internal jugular veins (IJV) according to the formula:

$\mathrm{V}$ max IJV optimal $=2 \mathrm{~S} \mathrm{CCA} \times$ Vps CCA/ $3 \mathrm{~S} \mathrm{IJV}$

IAVR = max IJV actual $/ \mathrm{V} \max I J V$ optimal $\times 100 \%$, where

IAVR - index of arteriovenous ratio, (\%)

Vps CCA - peak systolic velocity of the CCA, $\mathrm{cm} / \mathrm{s}$

Vmax IJV - maximum blood flow velocity, $\mathrm{cm} / \mathrm{s}$.

$\mathrm{S}$ - vessel cross-sectional area, $\mathrm{cm}^{2}$, as well as.

To assess the neurological status of patients, rating scales were used, including a visually analog headache intensity scale.

Results: The greatest prognostic significance was possessed by such parameters as the indicator of arteriovenous blood flow ratio and the value of headache intensity on a visual-analogue scale. A model for the differential diagnosis of AIS and VIS, which has high specificity and sensitivity, has been developed. Velocity indicators in the veins of Rosenthal and the vein of Galen are additional signs of intracranial venous stasis.

\section{Keywords}

Arterial ischemic stroke (AIS), Venous ischemic stroke (VIS), Cerebral 
venous sinus thrombosis, Ultrasound index of arteriovenous ratio (IAVR), Visual analog headache intensity scale

\section{List of Abbreviations}

AIS: Arterial Ischemic Stroke; VIS: Venous Ischemic Stroke; IJV: Internal Jugular Veins; MSCT: Multispiral Computed Tomography; MSCTA: Multispiral Computed Tomographic Angiography; CCA: Common Carotid Artery; IAVR: Ultrasound Indicator of Arteriovenous Ratio of Brachiocephalic Vessels; PCT: Perfusion Computed Tomography; NIHSS: National Institutes of Health Stroke Scale; VPS: Velocity Peak Systolic

\section{Introduction}

The study of ischemic stroke remains one of the priority areas of neurology because of its prevalence and severity of consequences. Despite the fact that at present, arterial stroke is quite well studied, approaches to the diagnosis and treatment of $22-30 \%$ of cases in its classification are developed and systematized for cryptogenic stroke [1]. Indeed, the etiology of a stroke cannot always be determined, and the cause of its occurrence remains unknown [2-4]. Generally, the term "stroke" refers to cerebral artery disease. At the same time, with the pathology of cerebral venous structures, the formation of a focus of ischemia is also possible, but this happens relatively less often and has been studied to a much lesser extent.

It is known that venous stroke (VIS) accounts for $0.5-1 \%$ of all ischemic strokes [5]. Strokes associated with cerebral venous sinus thrombosis are prone to secondary hemorrhages in $40 \%$ of cases, which is significantly more likely than arterial $[6,7]$ and due to the severity of vasogenic edema in VIS [8, 9]. It is important that the main approach to the treatment of VIS, which is more prone to secondary hemorrhage [10], is anticoagulant therapy, the safety of which in this group of patients has not been completely determined $[11,12]$. It is known that even with a favorable outcome within 6 months, approximately $30 \%$ of patients experience the development of chronic headache in the presence of hemorrhagic transformation. According to the results of a three-year followup, the number of patients with chronic headache increases to $60 \%[13,14]$. The complexity of the urgent diagnosis of VIS lies in often absence of specific clinical manifestations [15-17]. However, the most common occurrence of severe headache. This symptom occurs among patients' complaints in approximately $75-90 \%$ of cases [18-21]. In $25 \%$ of cases, headache is the only complaint of the patient; often, there is no connection between the localization of the headache and the site of sinus thrombosis [7]. Moreover, it can imitate migraine [22-24]. Early diagnosis of the relationship of headache with cerebral venous sinus thrombosis, even in the absence of neurological signs, is crucial for the timely detection of the disease $[25,26]$. At the same time, headache is an extremely common symptom, and the vast majority of patients with isolated headache do not have cerebral venous sinus thrombosis [27], which is why the economic feasibility of routine imaging remains very uncertain [28]. All this explains the need to search for new non-invasive and non-radiation accessible methods of emergency differential diagnosis of ischemic stroke of arterial and venous origin.

\section{Materials and Methods}

The study included 124 patients admitted to the clinic with non-hemorrhagic stroke from 2010 to 2013 and was in accordance with the ethical standards of the Helsinki Declaration and was approved by the Institute's Local Ethics Committee. The exclusion criteria were: aphasia, impaired consciousness, an anamnesis of stroke, hemorrhagic stroke, thrombolytic therapy, brain tumor, arterial aneurysms or other vascular malformations, the presence of stenoses of the brachiocephalic arteries more than 50\%, hemodynamically significant tortuosity of the carotid arteries, increased blood pressure above $130 / 90 \mathrm{~mm}$, jugular vein thrombosis, any cancer.

The study protocol included an assessment of the neurological status of the patient with used scales: National Institutes of Health Stroke Scale, Barthel, Rankin. A visual analogue scale was used to assess headache intensity. The patient himself evaluated his pain in the range from 0 (no pain) to 10 (the maximum possible pain). Patients underwent general clinical and laboratory examination. All patients underwent native multispiral computed tomography (MSCT) and multispiral perfusion computed tomography (PCT) for 40 minutes after admission to the hospital. Native MSCT was also performed on days 5-7 to detect secondary hemorrhage. Color duplex scanning of brachiocephalic vessels and transcranial duplex scanning were performed upon admission to the hospital, and the following were assessed: maximum blood flow velocity in the Galen vein and Rosenthal veins (a larger value of the blood flow velocity in one of the Rosenthal veins was taken into account, regardless of the side of the lesion).

The definition brachiocephalic vessels ultrasound index of arteriovenous ratio (IAVR) is based on the fact that the normal cross-sectional area of the internal jugular vein exceeds the cross-sectional area of the common carotid artery by 75$100 \%$. It is also known that the maximum blood flow velocity in the IJV is $1 / 3-1 / 2$ of the peak systolic blood flow velocity (VPS $\mathrm{cm} / \mathrm{s}$ ) by CCA. Knowing the cross-sectional areas of the IJV and CCA, as well as the peak systolic velocity of the CCA in each case, it is possible to determine the "optimal" speed from the IJV and compare it with the actual one using formulas (1) and (2) [29]:

$$
\mathrm{V}_{\max } \mathrm{IJV} \text { optimal }=2 \mathrm{~S} \mathrm{CCA} \times \text { VPS CCA } / 3 \mathrm{~S} \mathrm{IJV} \ldots \ldots(1)
$$

IAVR $=\max I J V$ actual $/ \mathrm{V} \max I J V$ optimal $\times 100 \%$, where........ (2)

IAVR - index of arteriovenous ratio, (\%)

VPS CCA - peak systolic velocity of the CCA, $\mathrm{cm} / \mathrm{s}$

$\mathrm{V}_{\max } \mathrm{IJV}$ - maximum blood flow velocity, $\mathrm{cm} / \mathrm{s}$.

$\mathrm{S}$ - vessel cross-sectional area, $\mathrm{cm}^{2}$. 
During the hospital period, the outcome of the disease was evaluated. A favorable outcome was the presence of positive dynamics in clinical status or the absence of negative dynamics (based on a dynamic assessment by a neurologist and the use of rating scales).

Statistical processing of the material was carried out using the STATISTICA 9.0 software package (StatSoft, Inc. www. statsoft.com). Comparison of quantitative traits in groups was performed using the Mann - Whitney and Kruskal-Wallis test. When assessing qualitative characteristics, the Pearson $\chi 2$ criterion with Yates correction was used. Differences were accepted as statistically significant at $\mathrm{p}<0.05$.

To develop a prognostic model for the differential diagnosis of VIS and AIS, a regression analysis in the form of binary logistic regression was used. The quality of the model was assessed by constructing a classification matrix. (Statistical Package for the Social Sciences program). Validation of this model was carried out on a sample of newly recruited patients (44 people), of whom nine had VIS and 35 had AIS. During validation, the model correctly classified VIS in $77.8 \%$ of cases, AIS in $94.29 \%$ of cases.

\section{Results}

The examined patients are divided into two groups based on the genesis of the stroke (venous and arterial). Comparative characteristics of the studied groups of patients are presented in table 1.

Analyzing the data obtained, we can conclude that, despite the absence of statistically significant differences in the main clinical and demographic indicators, patients with VIS were younger in comparison with patients with AIS, less likely to suffer from arterial hypertension and diabetes mellitus, they were more likely to have a favorable outcome.

In case of VIS, a characteristic symptom was a headache, as well as signs of cerebral venous stasis according to ultrasound methods, which are clearly demonstrated by such indicators as enlarged of maximum blood flow velocity in the Rosenthal veins and Galen vein as well as IAVR decreasing.

To select indicators that have the greatest prognostic ability in the differential diagnosis of VIS and AIS, we used a regression analysis in the form of binary logistic regression. The variable $Y$ was used as a dependent variable, characterizing the genesis of a stroke and assuming the value $Y=1$ in the case of AIS and $Y=0$ in the presence of AIS. The following factors were selected as a group of factors presumably predictive in diagnosing the genesis of stroke: the patient's age, manifestation of the disease, the values of headache intensity on admission, IAVR of brachiocephalic vessels, maximum blood flow velocity in Rosenthal veins and Galen vein.

Regression analysis left the headache intensity and ultrasonic IAVR of blood flow as the factors influencing the probability of the patient having VIS. Both factors had a significant impact on the assessment of the predictive probability of the presence of VIS. The higher the headache intensity and the lower the IAVR value of blood flow, the
Table 1: Comparative characteristics of the studied groups of patients according to the main clinical and demographic, laboratory, as well as ultrasound indicators.

\begin{tabular}{|l|l|l|l|}
\hline \multirow{2}{*}{ Indicators } & \multicolumn{2}{|l|}{ Stroke } & \multirow{2}{*}{$\mathrm{P}$} \\
\cline { 2 - 4 } & Venous $(\mathrm{n}=22)$ & Arterial $(\mathrm{n}=102)$ & \\
\hline Age (years), Me (Q25; Q75) & $57(43 ; 63)$ & $71(59 ; 77)$ & 0.2 \\
\hline Gender, male / female, $\mathrm{n}(\%)$ & $11(59) / 11(50)$ & $52(51) / 50(49)$ & 0.9 \\
\hline Arterial hypertension, $\mathrm{n}(\%)$ & $16(72.7)$ & $89(87.3)$ & 0.2 \\
\hline $\begin{array}{l}\text { Anamnesis of transient } \\
\text { ischemic attack, } \mathrm{n}(\%)\end{array}$ & $1(4.5)$ & $2(2)$ & 0.9 \\
\hline Diabetes, $\mathrm{n}(\%)$ & $1(4.5)$ & $11(10.8)$ & 0.6 \\
\hline $\begin{array}{l}\text { Visual analogue scale of } \\
\text { headache intensity (points), Me } \\
\text { (Q25; Q75) }\end{array}$ & $8(6 ; 8)$ & $3(2 ; 4)$ & 0.001 \\
\hline NIHSS (points),Me (Q25; Q75) & $9(2 ; 21)$ & $9(6 ; 14)$ & 1 \\
\hline Barthel Index, Me (Q25; Q75) & $35(0 ; 60)$ & $45(15 ; 65)$ & 0.2 \\
\hline $\begin{array}{l}\text { Rankin Scale (points), Me } \\
\text { (Q25; Q75) }\end{array}$ & $4(3 ; 4)$ & $4(3 ; 5)$ & 0.7 \\
\hline Hypercoagulation, $\mathrm{n}(\%)$ & $10(45.5)$ & $25(24.5)$ & 0.11 \\
\hline Hypocoagulation, $\mathrm{n}(\%)$ & $1(4.5)$ & $4(3.9)$ & 0.7 \\
\hline Favorable outcome, $\mathrm{n}(\%)$ & $20(90.9)$ & $86(84.3)$ & 0.6 \\
\hline $\begin{array}{l}\text { The maximum blood flowvelocity } \\
\text { in the vein of Rosenthal(cm/s), } \\
\text { Me (Q25; Q75) }\end{array}$ & $25(24 ; 33)$ & $15(10 ; 18)$ & 0.001 \\
\hline $\begin{array}{l}\text { The maximum blood flow } \\
\text { velocity in the vein of Galen } \\
\text { (cm/s), Me (Q25; } \mathrm{Q} 75)\end{array}$ & $33(30 ; 35)$ & $18(13 ; 25)$ & 0.0002 \\
\hline IAVR (\%), Me (Q25; Q75) & $45(23 ; 52)$ & $70(68 ; 73)$ & 0.00001 \\
\hline
\end{tabular}

more likely the patient will be diagnosed with VIS associated with cerebral venous sinus thrombosis. Using regression coefficients, for each patient, according to the measured values of indicators, the predicted probability of having VIS in accordance with the formula (3) can be calculated

$$
P(Y=1 / X 1, X 2)=\frac{1}{1+e^{-(4,626+0,856 \cdot X 1-0,192 \cdot X 2)}}
$$

where $\mathrm{P}$ - is the forecast probability;

e - the base of the natural logarithm (mathematical constant);

$\mathrm{X} 1$ - headache intensity value on a visual analogue scale at admission;

\section{$\mathrm{X} 2$ - IAVR, \%.}

The probability value varies from zero to 1 . If the predited probability is greater than 0.5 , then the patient will be diagnosed with VIS. If the probability value is less than 0.5 , then the patient will be diagnosed with AIS. Using the classification matrix, it was determined that the constructed model 98\% correctly classifies patients with AIS and 95.2\% correctly identifies VIS patients. The values of these indicators are high, and this means that the constructed model has a high prognostic ability (Table 2).

The volumes of the training and independent samples 


Table 2: Classification matrix for patients with venous and arterial strokes.
\begin{tabular}{|l|c|c|c|}
\hline $\begin{array}{l}\text { Supposed Stroke } \\
\text { Diagnosis }\end{array}$ & \multicolumn{2}{|l|}{ Established stroke diagnosis } & $\begin{array}{l}\text { Percentage correct } \\
\text { classifications }\end{array}$ \\
\cline { 2 - 4 } & Arterial & Venous & \\
\hline Arterial & 99 & 2 & 98 \\
\hline Venous & 1 & 20 & 95.2 \\
\hline
\end{tabular}

arwhich is due to the small number of cases of venous strokes due to the rarity of the disease. The quality of the developed model will be refined as data on patients with VIS are accumulated.

\section{Discussion}

Using the standard approach for the urgent diagnosis of stroke does not allow to detect ischemic stroke of venous origin. MSCT allows to detect cerebral venous thrombosis, PCT can provide additional information for the differential diagnosis of the genesis of stroke, however, these methods cannot be used in emergency diagnosis in all cases of acute ischemic stroke. Obviously, to improve the quality of the diagnosis of stroke, additional clinical and instrumental methods are required, the use of which, on the one hand, would not lead to a loss of time required to implement the standard protocol for examining urgent patients with acute cerebrovascular accident, and on the other, it would not additional significant financial costs. According to the data obtained, a decrease in the IAVR of the blood flow of the brachiocephalic vessels $50 \%$ and lower in combination with an increase in the intensity of the headache according to visual score data of more than 4 points is in favor of the VIS, thus, the prognostic model for the differential diagnosis of stroke allows one to suspect its venous genesis even before the use of neuroimaging methods, being the rationale for the appointment of MSCT with the mandatory conduct of the venous or equilibrium phase of the scan, as well as PCT. Indirect signs of intracranial venous stasis are elevated maximum blood flow velocity in one or both Rosenthal veins of more than $25 \mathrm{~cm} / \mathrm{s}$ and Galen's vein above $30 \mathrm{~cm} / \mathrm{s}$, obtained by transcranial duplex scanning.

\section{Conclusion}

Current standards for the differential diagnosis of stroke do not allow early detection of patients with VIS with sufficient effectiveness. The study made it possible to identify additional signs of intracranial venous stasis, the detection of which is not associated with radiation exposure and does not require the introduction of an iodine-containing contrast medium. It is noteworthy that the use of non-invasive (including ultrasound) techniques in the AHA/ASA 2018 recommendations is considered preferable in cases where the subsequent implementation of mechanical thromboextraction is not implied, while the use of multimodal (using MSCT and MRI) approach to the diagnosis of stroke is not recommended due to a possible delay in the decision to perform thrombolytic therapy (Class III, level of evidence B, randomized trials is no exist) [30]. Assessment of velocity parameters in the Galen vein and Rosenthal veins during transcranial scanning is an additional indirect sign of intracranial venous stasis, but the use of the method is limited by the absence of an acoustic window according to various data in $10-20 \%$ of cases [3133]. The use of ultrasonic IAVR (within the protocol of color duplex scanning of brachiocephalic vessels), as well as the assessment of headache intensity according to the visual analogue scale in the developed model, improve the quality of early diagnosis of acute ischemic stroke and get the predicted likelihood of a patient having a venous stroke without the need for the cumbersome formula presented above. It should be noted that when using the visual analogue scale of headache intensity, there are a number of limitations: the subjectivity of its assessment, the presence of aphasia, as well as impaired consciousness in a patient with a stroke, while IAVR is an easy-to-use accessible parameter that can make a significant contribution to diagnosis of VIS.

\section{Conflict of Interest Statement}

The authors declare that the research was conducted in the absence of any financial, commercial or other relationships that may be perceived by the academic community as representing a potential conflict of interest.

\section{References}

1. Arauz A, Merlos-Benítez M, Roa LF, Hernández-Curiel B, Cantú C, et al. 2011. Cryptogenic stroke in young patients: long-term prognosis and recurrence. Neurologia 26(5): 279-284. https://doi.org/10.1016/j. nrl.2010.10.010

2. He D, Shi Q, Xu G, Hu Z, Li X, et al. 2018. Clinical and infarction patterns of $\mathrm{PFO}$-related cryptogenic strokes and a prediction model. Ann Clin Transl Neurol 5(11): 1323-1337. https://doi.org/10.1002/ acn3.647

3. Fonseca AC, Ferro JM. 2015. Cryptogenic stroke. Eur J Neurol 22(4): 618-623. https://doi.org/10.1111/ene.12673

4. Smer A, Salih M, Mahfood Haddad T, Guddeti R, Saadi A, et al. 2018. Meta-analysis of randomized controlled trials on patent foramen ovale closure versus medical therapy for secondary prevention of cryptogenic stroke. Am J Cardiol 121(11): 1393-1399. https://doi.org/10.1016/j. amjcard.2018.02.021

5. Garland J, Kesha K, Vertes D, Modahl L, Milne D, et al. 2018. Empty delta sign on unenhanced postmortem computed tomography scan in cerebral venous thrombosis. Am J Forensic Med Pathol 39(4): 360-363. https://doi.org/10.1097/PAF.0000000000000421

6. Bonneville F. 2014. Imaging of cerebral venous thrombosis. Diagn Interv Imaging 95(12): 1145-1150. https://doi.org/10.1016/j.diii.2014.10.006

7. Wasay M, Kojan S, Dai AI, Bobustuc G, Sheikh Z. 2010. Headache in cerebral venous thrombosis: incidence, pattern and location in 200 consecutive patients. J Headache Pain 11(2): 137-139. https://doi. org/10.1007/s10194-010-0186-3

8. Mendel TA, Błażejewska-Hyżorek B, Szpak GM, Stępień T, Lewandowska E, et al. 2017. Intracerebral hemorrhage in the context of cerebral amyloid angiopathy and varied time of onset of cerebral venous thrombosis: a case report. Folia Neuropathol 55(3): 242-248. https://doi. org/10.5114/fn.2017.70490

9. Semenov S, Portnov Yu, Semenov A, Korotkevich A, Kokov A. 2017. Neuroimaging patterns of cerebral hyperperfusion. J Phy Conf Ser 886: 012014. http://doi.org/10.1088/1742-6596/886/1/012014

10. Kato Y, Takeda H, Furuya D, Nagoya H, Deguchi I, et al. 2010. Subarachnoid hemorrhage as the initial presentation of cerebral venous 
thrombosis. Intern Med 49(5): 467-470. https://doi.org/10.2169/ internalmedicine.49.2789

11. Tsai FY, Kostanian V, Rivera M, Lee KW, Chen CC, et al. 2007. Cerebral venous congestion as indication for thrombolytic treatment. Cardiovasc Intervent Radiol 30(4): 675-687. https://doi.org/10.1007/ s00270-007-9046-1

12. Viegas LD, Stolz E, Canhão P, Ferro JM. 2014. Systemic thrombolysis for cerebral venous and dural sinus thrombosis: a systematic review. Cerebrovasc Dis 37(1): 43-50. https://doi.org/10.1159/000356840

13. Breteau G, Mounier-Vehier F, Godefroy O, Gauvrit JY, MackowiakCordoliani MA, et al. 2003. Cerebral venous thrombosis 3-year clinical outcome in 55 consecutive patients. J Neurol 250(1): 29-35. https://doi. org/10.1007/s00415-003-0932-4

14. Busch MA, Hoffmann O, Einhäupl KM, Masuhr F. 2016. Outcome of heparin-treated patients with acute cerebral venous sinus thrombosis: influence of the temporal pattern of intracerebral haemorrhage. Eur J Neurol 23(9): 1387-1392. https://doi.org/10.1111/ene.13064

15. Gunes HN, Cokal BG, Guler SK, Yoldas TK, Malkan UY, et al. 2016. Clinical associations, biological risk factors and outcomes of cerebral venous sinus thrombosis. J Int Med Res 44(6): 1454-1461. https://doi. org/10.1177/0300060516664807

16. Mahale R, Mehta A, Varma RG, Hegde AS, Acharya PT, et al. 2017. Decompressive surgery in malignant cerebral venous sinus thrombosis: what predicts its outcome? J Thromb Thrombolysis 43(4): 530-539. https://doi.org/10.1007/s11239-017-1489-x

17. Semenov SE, Moldavskaia IV, Yurkevich EA, Shatokhina MG, Semenov AS. 2019. Diagnosis of venous ischemic stroke. Part I (clinical polymorphism). A review article. Complex Issues of Cardiovascular Diseases 8(2): 125-134. https://doi.org/10.17802/2306-1278-2019-82-125-134

18. Carangelo B, Lavalle L, Tiezzi G, Branco D, Lippa L, et al. 2015. A rare localization of cerebral venous sinus thrombosis: case report. G Chir 36(2): 79-83.

19. Alberti A, Venti M, Biagini S. 2008. Headache and cerebral vein and sinus thrombosis. Front Neurol Neurosci 23: 89-95. https://doi. org/10.1159/000111263

20. Benabu Y, Mark L, Daniel S, Glikstein R. 2009. Cerebral venous thrombosis presenting with subarachnoid hemorrhage. case report and review. Am J Emerg Med 27(1): 96-106. https://doi.org/10.1016/j. ajem.2008.01.021

21. Goyal G, Charan A, Singh R. 2018. Clinical presentation, neuroimaging findings, and predictors of brain parenchymal lesions in cerebral vein and dural sinus thrombosis: a retrospective study. Ann Indian Acad Neurol 21(3): 203-208. https://doi.org/10.4103/aian.AIAN_470_17
22. Alshurafa S, Alfilfil W, Alshurafa A, Alhashim K. 2018. Cerebral venous sinus thrombosis in a young female misdiagnosed as migraine ending in a permanent vegetative state: a case report and review of the literature. $J$ Med Case Rep 12(1): 323. https://doi.org/10.1186/s13256-018-1846-1

23. Karim OA, Dizdarevic A, Motawea M. 2017. Cerebral venous thrombosis misinterpreted as migraine. Ugeskr Laeger 179(11): V09160616.

24. Tan FU, Tellioglu S, Koc RS, Leventoglu A. 2015. Migraine-like headache in cerebral venous sinus thrombosis. Neurol Neurochir Pol 49(1): 78-80. https://doi.org/10.1016/j.pjnns.2015.01.005

25. Bushnell C, Saposnik G. 2014. Evaluation and management of cerebral venous thrombosis. Continuum (Minneap Minn) 20(2 Cerebrovascular Disease): 335-351. https://doi.org/10.1212/01. CON.0000446105.67173.a8

26. Sader N, de Lotbinière-Bassett M, Tso MK, Hamilton M. 2018. Management of venous sinus thrombosis. Neurosurg Clin N Am 29(4): 585-594. https://doi.org/10.1016/j.nec.2018.06.011

27. Chinthapalli K, Logan AM, Raj R, Nirmalananthan N. 2018. Assessment of acute headache in adults - what the general physician needs to know. Clin Med (Lond) 18(5): 422-427. https://doi. org/10.7861/clinmedicine.18-5-422

28. Saposnik G, Barinagarrementeria F, Brown RD Jr, Bushnell CD, Cucchiara B, et al. 2011. Diagnosis and management of cerebral venous thrombosis: a statement for healthcare professionals from the American Heart Association/American Stroke Association. Stroke 42(4): 11581192. https://doi.org/10.1161/STR.0b013e31820a8364

29. Shumilina MV. 2012. Integrated ultrasound diagnosis of peripheral vascular disease. A.N. Bakulev National Medical Research Center of Cardiovascular Surgery, Moscow, Russian Federation.

30. Powers WJ, Rabinstein AA, Ackerson T, Adeoye OM, Bambakidis NC, et al. 2018. 2018 Guidelines for the early management of patients with acute ischemic stroke: a guideline for healthcare professionals from the American Heart Association/American Stroke Association. Stroke 49(3): e46-e110. https://doi.org/10.1161/STR.0000000000000158

31. Director LT, Mackenzie DC. 2018. Dural sinus thrombosis identified by point-of-care ultrasound. Clin Exp Emerg Med 5(3): 199-203. https://doi.org/10.15441/ceem.17.237

32. Purkayastha S, Sorond F. 2012. Transcranial Doppler ultrasound: technique and application. Semin Neurol 32(4): 411-420. https://doi. org/10.1055/s-0032-1331812

33. Zamboni P. 2016. Why current Doppler ultrasound methodology is inaccurate in assessing cerebral venous return: the alternative of the ultrasonic jugular venous pulse. Behav Neurol 2016: 7082856. https:// doi.org/10.1155/2016/7082856 